\title{
ON LANGUAGE USE AND SOCIAL STRUCTURE
}

\author{
REID LUHMAN \\ University of Kansas
}

\begin{abstract}
The emerging field of sociolinguistics is a response to numerous roadblocks encountered in the more specific area of linguistics. One of the more important of these roadblocks occurred in attempts to provide a linguistic explanation of bilingualism through interpreting languages in contact in terms of an interference perspective. Such a perspective emphasizes structural aspects of languages as explanations of changes in either (or any) language within the contact situation. The language contact situation, however, made extremely evident that explanations of language use must include social factors. In the case of bilingualism, the more general sociolinguistic perspective emphasizes inter-relations between language use and socially constructed situations at the micro level. At the micro level, language forms can be viewed as tools with which social meanings are constructed and communicated, each utterance thereby containing an information aspect (which is obvious) and a more general social aspect. At the macro level, language forms become markers of the relations between and among complex social groups and, in this sense, reflect the more purely sociological concerns of class and stratification. The upshot of this new perspective is that all utterances come to be viewed as tools and containers of social meaning regardless of whether those utterances come from one recognized language or from six recognized languages; people use their sounds to discriminate meaning and will accomplish that discrimination with whatever system they have at hand. Hence, through a sociolinguistic perspective, bilingualism becomes but a special case of this process.
\end{abstract}

The purpose of this discussion is to illustrate some relationships between language forms and social structure in such a way that the former may be used in understanding the latter. The core assertions in this discussion are two: (1) That language forms are tools with which social structure is made sensible to members, and through which it is constantly re-created by members; and (2) that those language forms can be made sensible to the observer and used as indices of that created social structure.

In the course of developing these two assertions, the organization of this discussion will reflect two highly related changes in assumptions in the field of language concerns: (1) The assumption that recognized languages are inviolable rule systems from which all speech forms are derived has been altered to account for rules governing those speech forms labeled "creole," "dialect," and "style;" and, (2) The assumption that competence in a language can be isolated from the ability to perform through speech (Chomsky, 1965) has been altered in an attempt to integrate language use and ability with its social contexts. Both of these assumptions were brought to bear upon the earlier studies of bilingualism and both came to be questioned as the social contexts of bilingualism became increasingly important in explaining that phenomenon. The changes in these assumptions laid the groundwork for a sociology of language. This discussion will begin with those earlier studies in bilingualism, will then trace some of the conflicts that arose, and finally will outline a perspective integrating the results of those conflicts with the social contexts of speaking. 


\section{Language Interference}

If a single theoretical issue can be isolated in the field of linguistics as the gadfly responsible for bringing language concerns under the rubric of sociological and anthropological perspectives, it would have to be the problem of understanding bilingualism. The "pure" language studies based on one informant speaking in a laboratory were derived from the assumption that there was a one-to-one correspondence between culture and language. One culture with one language requires a sample of only one person. This assumption was absolutely unworkable in a bilingual situation, however, due to the blatant variety in language forms encountered. Nevertheless, while linguists involved with bilingual studies came to the realization that language varied with and was often dependent upon social organization, their research was built upon the basic langue-parole distinction of deSaussure, and this, to a large extent, determined the kinds of results they obtained.

DeSaussure's distinction concerns the basic structure or rule system (langue) of language on the one hand, and the ability of any individual to speak it (parole) on the other. The point of this distinction is that language is an internally coherent body of rules for communication and, as such a body, should be conceptually abstracted from actual forms of communication. The assumption continues that "speakers" of a language are those who possess an understanding of and an ability to manipulate that body of rules in response to any particular situation requiring communication. The importance of this assumption, then, is that any given act of communication in any situation can be viewed as a logical (although sometimes incorrect) derivative of the rule system, this conclusion thus leaving the researcher justified in studying the latter without getting his hands dirty.

Extending this assumption to the study of bilingualism, the researcher is then confronted with two or more such "rule systems" being used concomitantly by the same individuals as the basis for their individual acts of communication. The question that arises is to what extent do those individual acts of communication maintain a separation between the two rule systems and to what extent do they combine aspects of both rule systems through producing sentences which have no single basis in either language? The former case is called "pure bilingualism" and the latter case is called "interference." Research along this theoretical continuum has been the basis for almost all "linguistic" efforts in bilingualism and, until recently, has been viewed as an end in itself (LePage, 1969: 142-3). Nevertheless, whether against its will or not, the perspective of interference has become a means to other ends, and for this reason, it seems valid to take a closer look at its development.

The modern classic work in interference is Uriel Weinreich's Languages in Contact, first published in 1953. He defines interference as "the rearrangement of patterns that result from the introduction of foreign elements into the more highly structured domains of language, such as the bulk of the phonemic system, a large part of the morphology and syntax, and some areas of the vocabulary" (Weinreich, 1953: 1). Interference is "the rearrangement of patterns" and clearly refers to language as a rule system. As Weinreich points outs, the notion of interference assumes that every speech event belongs to a specific language (Weinreich, 1953: 7). With this as a starting point, Weinreich proceeds to subdivide the rule system of language and makes an effort to isolate and explain every aspect of interference that might occur within each subdivision. But before following the story of interference any further, it might be well to take a quick glance at the last page of the narrative and note that a later linguist, Nils Hasselmo, defines interference (in part) as "the simultaneous application of the patterns of 
two languages to the same item" (Hasselmo, 1969: 123). The "patterns" of Weinreich have become the "application of the patterns" for Hasselmo, and the study of interference is clearly going to confront DeSaussure's "parole" before it's through.

Returning to the breakdown of language for the purpose of isolating interference, probably the basic starting point would be the morpheme. The morpheme is the smallest unit which communicates grammatical relations-a definition which includes the communication of meaning (Liles, 1972: 142). It accomplishes this in part through systematically following phonological rules. This suggests that any study of interference would have to be concerned with both the morphemic and the phonemic aspects of language, and this is precisely the position of Einar Haugen (1956). Weinreich chose to divide the morphemic analysis into two categories-grammatical relations and lexicon (Weinreich, 1953). Such a distinction (although redundant for Haugen) is of particular utility for sociological concerns in that it permits a separate analysis of language's designative function as it structures the environment into a sensible realm for the speaker.

Viewing the phonemic aspects of language from an interference perspective has replaced the common sense notion of mispronunciation. Sounds that come from speakers are usually as systematically "incorrect" as they are "correct." In a bilingual situation, the fundamental question becomes one of contrasting available phonemes in both languages. The problem for the speaker of reproducing a sound he hears in another language or even hearing the sound in the first place may be largely determined by the sounds he is accustomed to discriminating and reproducing. Beyond this, the act of learning a "second" language's phonemes (to whatever degree) may in turn influence the phonemes he uses in his "first" language. Of all reflexive types of interference (from language two to language one), there is generally the least amount at the phonemic level and, as follows, the "accent" is usually the last to go in learning the second language. Rayfield (1970) suggests that this is due to the stage of life when learned; that the random sounds of a child become shaped into the standard phonemic forms of the language in his environment well before he produces words or sentences in that language. In addition, the "sounds" of a language are far less conscious to the speaker than are words or grammar.

For the bilingual individual, the experience is one of maintaining two sets of sounds with those respective languages. As is commonly the case, the two sets of sounds will have some degree of overlap when pronounced "correctly" and understandably may come to have more overlap depending upon an individual's confrontations with the languages in the contact situation. Such phonemic interference then becomes a rather effective marker of the form and degree of those confrontations for the speaker's audience.

At the level of grammatical relations occur those rules concerning sequence, agreement, intonation, dependence, and so on. All aspects of grammar are basic to communication and, like phonemes, can become altered in the contact situation. Once again, grammatical relations can be borrowed directly into a language or can have the indirect effect of altering the structure and/or meaning of those forms originally common to that language (Weinreich, 1953: 30). According to Weinreich, this sort of interference will be directly related to the structural independence of the grammatical form, the more independent, the more easily borrowed (Weinreich, 1953: 36). It is also informative of the borrowing language. When a verb, for instance, is borrowed but is conjugated in one of several alternative forms of the borrowing language, that form selected suggests something about perceptions of rules or regularities among the speakers concerning their own grammar. In short, the level of 
grammatical relations of a language is subject to a unique form of interference, but like the phonemic level, it also marks the language (and, of course, social) confrontations of the speakers.

According to Weinreich's notion of structural independence of morphemes, the lexicon of a language is clearly the most open to borrowing and, at least in terms of absolute frequency, this is the case. The relationship between the lexicon and the other structural levels of language leads to particular kinds of lexical interference. The two possibilities of either outright borrowing from language two or redefining already existing structures in language one that have run throughout the whole discussion of interference more clearly illustrate the relation of language to social meaning and point out the limitations of a purely interference orientation insofar as it ignores the social dimension of speech.

The borrowing of single object words is one of the most common forms of lexical interference. In Colorado Spanish, for instance, the words "troca" (truck) and "torque" (turkey) have been clearly borrowed and spelled in Spanish in such a way as to most closely approximate the English pronunciation (Weinreich, 1953: 48). An example of redefining existing structures occurs in the same dialect with the Spanish word "ministro" (cabinet official, in Spanish) which has come to refer to Protestant ministers as well (Weinreich, 1953: 48). The sounds of new words are also a factor in redefinition, as the Portuguese word "pinchar" (to jump) has come to mean "to pinch" in American dialects of Portuguese (Weinreich, 1953: 49). In addition, there are induced creations such as the German word "Wolkenkratzer" (cloud scratcher literally) based on the English word "skyscraper," and hybrid creations, such as the Florida Spanish "home plato" (home plate, in baseball) using "home" from English and "plato" from Spanish (Haugen, 1958: 783; Weinreich, 1953: 52). It should be obvious that there is an interplay between all structural levels of language and the social dimension of meaning, but perhaps this point can be made through one additional example.

In American Italian exists the borrowed word "giobba" based on the English word "job" constructed in a similar manner to the "torque" example above. It was assumed that perhaps this new word was simply a synonym for arte, mestiere, professione, impiego, or occupazione, all of which have some bearing in Standard Italian to employment. In checking out this assumption, however, it was found that Italian Americans who used the word defined as "work that is found and for which one has no attachment and no spiritual interest" (Weinreich, 1953: 54). They stated further that only in America was such a word necessary and that there were no Italian words which captured the meaning. Accepting this definition, the two closest Italian words, "impiego" (employment) and "lavoro" (work), became somewhat restricted in their meaning as giobba took over some of their possible applications. The point, of course, is that words are learned and, in the bilingual situation, borrowed in terms of a social context. The importance of this borrowing is therefore of direct relevance not only in terms of pure language interests or as indicators of social structure, but also in terms of how individuals come to perceive their social environment and their relation to it. Giobba was clearly learned and associated with a significant feature of the Italian experience in this country.

\section{Language Dominance and Semantic Structure}

To further set the stage for both the changing assumptions in linguistics and for the increasing relevance of linguistic concerns for sociology, it would be useful to look at some of the research concerning bilingualism, semantic structure, and 
verbal ability. This research has largely been the domain of social psychology and has had numerous repercussions on language policy, particularly with regard to education.

Weinreich (1953: 9-10) attempted to account for dual language learning and semantic structure through the following schematic processes:

(A)

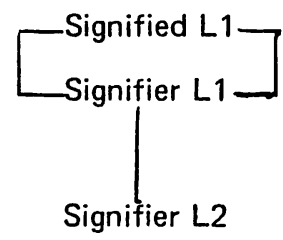

(B)

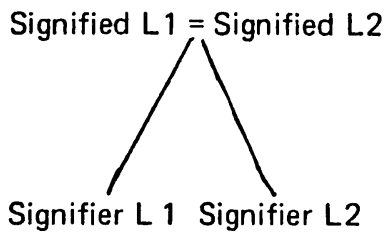

(C)

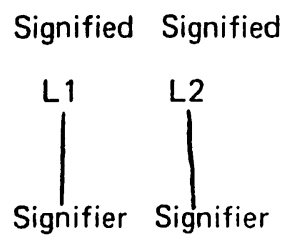

L1

[ $L 1=$ Language one and $L 2=$ Language two]

Diagram (A) depicts an initial confrontation with a new language. It is assumed that within language one, the signified (idea/word) and the signifier (sound which represents it) are combined as one unit. Language two is then learned insofar as its signifiers can be organized with those units in language one. Diagram (B) represents a more "fluent" situation, but one in which there is an equality (or direct translation potential) between both signifieds and signifiers. An individual represented by this scheme would have access to two languages as rule systems of communication but would be operating with essentially one semantic structure with which both rule systems were connected. Diagram (C) describes a "pure bilingual" operating with two rule systems, each with its own system of semantic meaning. This individual could be very fluent in both languages, but would also view them as completely different activities in terms of how they structured the world. As follows, this individual would also be a very poor translator as he would be lacking any means for forming equivalences between the languages.

While Weinreich mentioned this in passing, these ideas were formalized and more elegantly packaged by Ervin and Osgood (1954) with their compound-co-ordinate distinction. Compound bilingualism is essentially diagram (B) and co-ordinate bilingualism is essentially diagram (C). They conceived of their concepts as ideal types located on either ends of a continuum and as representing the variety of mental sets that a bilingual might possess. By and large, it would be difficult to notice the difference between the two through speech except that a compound bilingual would be far more vulnerable to interference since his distinction between the languages was more at the conscious level as two sets of rules learned as requiring separation in use. More importantly, however, Ervin and Osgood maintained that the true test was that of translation. A compound bilingual would have little trouble whereas a co-ordinate bilingual would have to go through all manner of machinations and could still not match the compound. Translation, Ervin and Osgood suggest, is learned like anything else, and the experience of a co-ordinate bilingual in translation activities would soon turn him into a compound bilingual. While this information on bilingualism could be important in itself to someone hiring translators, the more significant aspect for sociology concerns the social contexts within which varying forms of bilingualism arise. As pointed out by Macnamara (1967), the co-ordinate bilingual exists because he acquired his languages in different social contexts. As with 
an individual word such as giobba, so also are languages subject to their contexts, and parallel social contexts lif one can create such a metaphorical abstraction) would result in parallel semantic systems, each semantic system tied tightly to whatever language was used in its context (see Haugen, 1965).

A further derivative of the inviolable language assumption is the theory of language dominance. The theory of dominance assumes that since languages are separate systems, one of them is going to be the more (or most) dominant. An extension of this is termed the balance effect and it describes language acquisition and use as a zero-sum game in which the greater ability an individual has with one language, the lesser ability he will have in any other (Macnamara, 1966: 15-16). This theory goes on to state that the more two languages are structurally dissimilar, the more difficulty the bilingual individual will have with both. The basic assumption behind this theory, outside of assuming that languages are fixed systems, seems to be a time factor. An individual spends only so much time in language-related activities, and the more time he spends in one language, the less time he will spend using another. Although it is freely admitted that such an assumption is only really valid in reference to lexicon (as opposed to grammatical structure, for instance, which constantly repeats itself in use), this perspective has directed numerous studies into bilingual ability and has had a fundamental effect upon compulsory monolingualism in education.

In measurement, the balance theory is reflected in a number of tests designed to determine degree of bilingualism. Following a structural breakdown of language into semantics, syntax, lexicon and phonemes (or graphemes, if reading), language ability is measured across another dimension consisting of speaking, writing, listening and reading (Macnamara, 1969: 81). Of the many language tests, those most closely associated with the balance theory focus on time (as a measure of fluency) and ambiguity (as a measure of dominance) (Macnamara, 1967: 62-64; 1969: 86-89). The time focus assumes that quickness in all the language structural and use categories mentioned above effectively measures fluency. The time assumption is, however, clearly an assumption and may or may not have any relation to the subjects being tested who may respond quite differently to time restrictions than a white middle-class researcher accustomed to a more stop-watched existence (Fishman, Cooper and Ma, 1971: 557). The ambiguity focus assumes that a bilingual speaker presented with a word which could be pronounced and/or interpreted in either of two languages will select that language which is dominant. This latter measure requires, of course, two relatively similar languages. In Spanish and English, for example, "debate," "social," and "control" are spelled the same and mean roughly the same things but are pronounced distinctively differently.

These kinds of tests coupled with assumptions about language balance have been much used with other measures of social class and intelligence in the educational field. Carrow (1957) found that San Antonio, Texas Chicanos who were supposedly bilingual spoke neither language "well" and simultaneously scored lower on nearly every language test when matched for age, social-economic status and non-verbal IQ measures with Anglo mono-linguals. Peal and Lambert (1962) found exactly the opposite with bilingual French-Canadians who consistently outmatched their monolingual counterparts in language ability tests (see Macnamara [1966] for a thorough review of this research). Other research exists with findings at every point in between the above two extremes, suggesting that the balance theory is not the best explanation for bilingual vs. monolingual language ability. This kind of research can, however, be of use to the sociologist for the very different end of identifying social contexts of language use. 
The beginning assumption concerning language ability measurement based on two dimensions, structure and media, provides a useful index for the observer of where language was learned, how it was learned, and how it is used on a day-to-day basis. An individual will not necessarily encounter reading, writing, listening, and speaking experience in a language in the same contexts. It is common, for instance, for an immigrant to a second language culture to master a listening ability, develop a workable speaking ability, and have no ability whatsoever in reading and writing. Conversely, a second language learned through an educational setting would foster a good reading and writing ability coupled with either an underdeveloped or overly formal speaking ability (Sawyer, 1971: 381). Beyond these relatively extreme examples, even most educated monolingual speakers in any culture will have definite strengths in one or another of the media forms of his language. Few of us, for instance, are both comfortable and accomplished public speakers, theoretical physics readers, or short story writers. The media forms of any language with their respective subdivisions provide excellent indices of social experience based on the social contexts within which those particular forms are emphasized and, more importantly, necessary for the participants to learn.

Perhaps the real strength of an interference perspective (which focuses on the other dimension mentioned above; that of the structural components of a language) lies in its merging with the media perspective. Both media forms of a language and its structural components (grammar, lexicon, etc.) are functions of speaker contact with language situations. The notion of interference focuses primarily on only the one dimension and only on situations of language contact. Generalizing beyond situations of clearly recognized language contact and adding the second dimension of media provides a perspective both more useful in language contact situations as well as allowing a comparison between those situations and others with perhaps only dialect or style variation across the speech community. In this sense, then, it would be possible to differentiate a speaker's grammar, lexicon, and pronunciation within each media form, the composite picture being one index of that speaker's position within a social structure. The assumption here is that the stratification of social structure is essentially a matter of differential access to resources, power, etc., and that these access routes are marked and maintained symbolically through a parallel differential access to the symbols. Of these symbols, language use is certainly one of the most important.

At this point in the discussion, there are clearly two major theoretical obstacles in the path. First, there is the question of how language can be a coherent meaningful marker of social membership. Beyond the question of the speaker's conscious goals of communicating information, how in addition is it possible for that same utterance to communicate a wide range of social meaning, both intentional and unintentional on the part of the speaker? The first of these obstacles can be approached through a discussion of languages as codes with rules of co-occurrence (the following two sections), and the second through a discussion of the socially defined language domain (the final section).

\section{Languages as Codes}

A code is the process of converting meaning into signs and back into meaning according to rules. The conversion into signs is encoding and the conversion back into meaning is decoding. Both of these processes are directed by the rule system of the code so that, by knowing and following the rules, any individual can note the signs encoded by another and decode them 
back into more or less the original meaning. This definition is designed to be very general so that it might apply to the colorful waving of flags from the masts of ships as well as all normal use of any language among its speakers. Since, of course, language is the subject of this discussion, it might seem that the more general perspective of code is somewhat unnecessary; that it is one more attempt on the part of social scientists to remove their discourse from the realms of others. But this is not the intent here. The focus on all speech as code forms will not only result in a great economy of words but will also permit a much easier introduction of language concerns into the framework of sociological interpretation. The role of the code perspective in this theoretical relocation will be to initially call into question certain assumptions about language and its relation to other social processes.

Re-interpretations are usually applied to past assumptions and conclusions before they begin breaking new ground. Following in this tradition, a return to language interference with code perspective in hand results in a picture of the bilingual as one engaged in code-switching between one rule system and another without overlap (Hasselmo, 1969: 123). To the extent, then, that an individual switches in accordance with the phonological and morphological rules of each system, he may be said to be a "pure" bilingual. But once again, the assumption of the inviolable rule system is confronted. Consider, for example, the following quotation:

Once the local code is established, any divergence from it in the samples of speech of a bilingual as a result of the other language may be analyzed as a case of bilingual interference. (Mackey, 1965: 241)

In emphasizing both "local" and "code," Mackey is clearly aware that languages (like all codes) are arbitrary. He maintains that all notions of language must be empirically determined, and if the only way of expressing something in the local code is through a "borrowed" word (such as "bouquet" in English), then that word shall be considered to be part of the local code, no matter how recent the borrowing. One might well ask Mackey how he then knows when "the local code is established"? But he has already been "asked":

There is no such thing as a language except insofar as the verbal habits of two or more people overlap. It may therefore be misleading from the outset to speak of interference in the case of bilinguals possessing two sets of norms; interference occurs precisely because and to the extent that people are not bilingual and do not possess two sets of norms (LePage, 1969: 144).

Responses such as LePage's, directed to the shortcomings of the interference perspective are the result of his (and others') experiences in the field wherein assumptions regarding languages as separate and internally coherent systems quite simply do not work (see also Fishman, Cooper and Ma, 1971: 561-3).

One of the advantages of the code perspective is that it is far more open to being whittled down, when necessary, into sub-categories within recognized languages. This point is summed up very strictly by John Gumperz:

Rather than characterizing members as speaking particular languages it seems reasonable to speak of speech behavior in human groups as describable in terms of a linguistic repertoire consisting of a series of functionally related codes. Depending on the history of such communities, these codes may be dialects, styles, or superposed varieties of the same language or also genetically distinct languages. There is evidence to show that in such 
repertoires the same social determinants may be operative in situations of stylistic and bilingual selection. In other words, rules determining alternation between distinct languages or dialects may serve the same communicative ends as the selection of optional rules with a single language. These selection rules are not simply a matter of conscious choice. They operate in response to communicative contexts much like the grammatical choice, between, for example, present and past tense verb forms (Gumperz, 1972: 145) [my emphasis].

If it is possible to be even more succinct, one might add that whenever you find a social structure, you will also find people talking within it. The ways in which they label their talking may or may not be important, depending upon the questions with which the observer concerns himself. In any attempt to explain that social structure and language's symbolic importance within it, bilingualism is only a special case of that relation (Gumperz and Hymes, 1972: 38-9; Hymes, 1967: 9).

The concept of code is clearly moving in the direction of finding roots in the social environment of communication. In generalizing to account for the social ramifications of speech variation, a socially significant style shift by a monolingual speaker also becomes a significant code shift. The importance of this, as Labov (1972) has pointed out, is that there are no single style speakers. Hence, code-switching is a fundamental aspect of all communication. The "linguistic repertoire" of a speaker, as mentioned by Gumperz, may encompass a number of "different" languages in response to a communicative situation, or it may include only one recognized language. In the latter case, whatever communication distinctions might be made through language switching can be made just as clearly through code-switching within that language. In both cases, the form of communication is tailored to the social environment and reflects the structured variation of situations within that environment. Hence, previous distinctions among the concepts of language, dialect, creole, pidgen, argot (from deviant to respectable group forms), and style tend to soften somewhat as all these communication forms become viewed as codes susceptible to being switched into and out of. This "nest" of terms (which reflects that assumption about language mentioned earlier) also becomes less important as a group in that switching style to style, style (in one language) to dialect (in another), or language to language all are manifestations of code-switching of potentially equal importance. For example, a Chicano in the United States who switches between "pachuco" (a youth gang-oriented argot with elements of Spanish and English) and some variety of English is every bit as important from a code-switching perspective as a Chicano who switches between Standard Spanish and Standard English (Barker, 1950; 1947). The former case represents argot to dialect switching (perhaps) while the latter is clearly language to language switching. The former Chicano would likely be young, live in a big city barrio (probably in the Southwest), and more than likely be lacking both money and power, while the latter Chicano would just as likely be a member of one of the "old families" of the Southwest and have a relatively high social position in Anglo society coupled with a great deal of pride in the Spanish heritage (Barker, 1947; Christian and Christian, 1966). It is possible to know quite a bit about these two Chicanos as their two social positions present different linguistic repertoires to them, but as repertoires, both have considerable code variability within them and allow the speakers the same significant distinctions through code choice.

The code perspective sheds a new and critical light on theories of language dominance and balance as well as interference (Gumperz, 1967: 50). The assumption that a bilingual will always be at a linguistic disadvantage makes little sense if all monolinguals face similar problems of learning elaborate code variation within their one language. In this sense, a 
bilingual can be viewed as an individual who has spread this same variation across at least two recognized languages. And it is here that the focus of the language assumption sharpens. A bilingual switches codes in response to his relation to the social structure. It is therefore understandable that he would not have the same perspective on code switching within either of his two languages that a monolingual speaker of one or the other might have. For example, a Belgian businessman who speaks French at the office and Flemish in his home would not have the same code variation within French as a French businessman who needs to make the same distinctions within one language. On a language test, then, the Belgian would not appear to have the same "ability" with French as the monolingual, the Belgian's "errors" in that language forming in the vacuum left when he normally switches completely outside the language. He would be lacking, therefore, what might be termed an "intimate style" in French. One of Macnamara's (1967) Flexibility Tests includes phrases of the type, "he is drunk," with the request that the subject come up with as many different ways of saying the same thing as he can think of. Not only would such knowledge vary within any one language depending upon any one individual's socializing proclivities, it would also be knowledge that, in the case of the Beligian businessman, would co-occur with a switch to Flemish. Hence, he would do poorly on this aspect of the French language test (not to mention lacking corresponding knowledge of business activities in Flemish) and could be taken as a validation of the balance theory. If the same test were limited to those "styles" of French which correspond to his use of the language, however, he would likely do as well (or conceivably better) than the monolingual, as Peal and Lambert (1962) found with French Canadian bilinguals. The strength of the code perspective lies in its ability to illuminate the complex code-switching of the "monolingual" as the basis for assumptions regarding bilingual language ability. It accomplishes this through focusing on the social environment of all language use and the relation between that environment and those speech forms associated with it.

The implications outlined above are a response to the social factors which surround speech. An adequate sociology of language requires an attempt at such an integration. It is therefore not surprising that this turn was taken as concerns broadened in linguistics. There remains, however, the question of how one might recognize a code if standard institutionalized definitions of languages are not employed. The argument that a formal grammar approach misses much of the communicative nature of speech requires, in a sense, a new grammar which accounts for the unity between code forms and the social settings within which those forms become sensible for speakers. The best basis for such a new grammar would be the co-occurrence rules of speech which mark those codes.

\section{Rules of Co-occurrence and Interpretation}

The search for the co-occurrence rule of speech is a response to the question of how it is possible to tell when someone is speaking "differently." This difference could consist of either another language or an alternative style. While in the former case, the listener may sense the difference through an abrupt loss of comprehension, the latter will be highly comprehensible and, in fact, must be highly comprehensible to him if he is to function at all normally within any social context. Following Labov's (1972) point that there are no single style speakers, the corollary would be that there are no single style listeners if our speaker is to be appreciated. Should any individual be incapable of discerning those differences imbedded in his social 
code universe of interlocutors, he would most clearly be labeled a deviant to whatever extent matched his incapability. The ability to recognize code-switching is the ability to communicate; they are part of the same process. The code employed, its content, the context within which it is employed, and a share in the assumptive world within which this configuration is perceived-all of these, as a unit, make up communication. Consider the following example:
A: What is your name?
B: Well, let's say you might have thought you had something from before, but you haven't got it any more.
C: I'm going to call you Dean.

[from Laffal, 1965: 85]

Speaker B not only speaks grammatically, he is also capable of using fairly involved syntax. But he doesn't make sense. He is, in fact, an individual labeled schizophrenic in conversation with a doctor, and somehow this label allows us to make sense of his not making sense. Yet we really have little idea of why he doesn't make sense except to say that most people answer that question differently. This brings up two important questions: Why do most people answer that question differently, and once they do it, how do we recognize it as being different? Returning to Labov and a more basic question, we then have: How do people link styles (codes) with social situations and how do they recognize switches among the styles? As suggested above, the search for rules of co-occurrence is a response to this question.

Co-occurrence is another way of stating the word "pattern," and patterns of one sort or another tend to be the goals of scientists. This particular pattern is constructed from the total interplay between the structural components of language and social setting. In a formal sense, co-occurrence refers to the interplay among the structural components only, but as will be readily apparent, co-occurrence rules extend far beyond that formal structure.

There are two dimensions of language structural co-occurrence-the horizontal and the vertical (Ervin-Tripp, 1971: 38). Horizontal co-occurrence rules specify "relations between items sequentially in the discourse" (Ervin-Tripp, 1971: 38). This refers to the same level of language structure. Given a few items drawn from a lexicon, one would not expect to hear certain other words drawn from the same lexicon. For example,

"Good afternoon, Reverend. Grab yourself a chippendale and take a load off."

somehow does not appear to have come from any situation except humor. One does not tell a Reverend to "grab" something or to "take a load off," and one usually does not tell anyone to "grab" an expensive piece of furniture. The sentence alternates between one set of horizontal co-occurrence rules and another. Similarly, a single sentence could violate phonemic rules or structural rules in its course. Once an utterance selects certain elements from a level of language, it commits itself, in a sense, to remain consistent at each level. Such co-occurrence, however, is far more useful for interpretation when coupled with vertical co-occurrence rules.

Vertical co-occurrence refers to predictability across the structural levels of language. The selection of syntax might also determine lexical items and phonemic rules. Coupled with horizontal rules, that initial selection of syntax, lexical items and phonemic rules would also affect future choices at each of those levels. Ervin-Tripp's (1971) example illustrates the 
simultaneous breaking of rules across both dimensions:

"How's it going, Your Eminence? Centrifuging okay? Also, have you been analyzin' whatch'unnertook t'achieve?"

The choice, "How's it going," is casual in style, yet without the more common "goin." In addition, the technical term "centrifuge" is clearly not matched with "okay," the verb "analyze" would generally receive the more formal pronunciation in the present participle, and the phrase, "what you undertook to achieve," would generally co-occur with clearer articulation. In addition, horizontal rules are broken with the interspersing of slang and formal lexical items and with varying use of ellipsis in the syntax. In short, this utterance is a communicative monstrosity.

A more useful example for purposes of relating co-occurrence rules to the codes they mark is provided by Labov (1969: 252):

(1) He don't know nothin'.

(2) He doesn't have an inkling of the truth.

Sentence (1) couples the double negative with the "in" ending on "nothing" and simple syntax. It is consistent. Sentence (2) removes the double negative and this co-occurs with new lexical items, new pronunciation, and different syntax. It also is consistent. Once again, a violation makes the point stronger. Consider:

(3) He doesn't have no inklin' of de truth.

(4) He don't have no inkling of the truth.

The possibilities are, of course, almost endless. But the basic point is that neither sentence (3) nor (4) would be an expected utterance except, once again, with humor. Sentences (1) and (2), however, both would be expected as both of them are internally consistent; whenever "he doesn't have" is heard in English, the listener has definite expectations of just which forms of utterances may follow and which may not. Similarly, the same listener would have expectations following "he don't have no," but they would be very different expectations of grammar, lexical choice, and so on. In short, the listener is capable of recognizing a style (or code) shift through recognizing language forms as patterns and through associating those patterns with social contexts of speaking. In the particular cases of sentences (1) and (2), which are both clearly English, he would probably be surprised to hear them coming from the mouth of the same speaker. That surprise would be indicative of the fact that the listener is not a speaker of Black English, nor does he associate frequently with people accustomed to switching between Black English and Standard English. If he had never heard Black English, his comprehension might drop as well, and this code change would approach that of a change to a "foreign language" for him. At the other extreme, if the listener were accustomed to switching between those codes himself, his perception of the style change would be different both in degree and in content. He would not be as surprised and simultaneously would assign a different meaning to the change. Once again, the degree to which a code change is extreme is more a matter of the experience of the communicators rather than a matter of formal definitions concerning langauges, dialects, or styles. 
An emphasis on co-occurrence rules appears to carry the study of language use some distance from the formal grammarian. More accurately, however, it extends the boundaries of that endeavor. As John Gumperz points out,

... verbal interaction is always rule-governed, and secondly, ... the rules of verbal interaction go considerably beyond what we normally understand by grammatical rules. The general linguist who is concerned with grammar only works at a level of abstraction which covers but part of the verbal communication process. A generative grammar for English, for example, states the basic rules which underly the verbal performance of such socially diverse peoples as Midwestern Americans, speakers of Indian English, Australians, Liberians and many more. It need however not account for what we know to be the many differences in the linguistic performances of these individuals (Gumperz, 1969: 242).

A sociology of language would clearly go beyond the generative grammarian in its perspectives-this point is certainly not new to this discussion-but in asserting that all verbal interaction is rule governed and that grammar rules do not extend as explanations of all verbal interaction, the result is that the concept of co-occurence rules must begin taking its name seriously. Those rules which are defined at the recognized language level as grammar must be replicated with the addition of a social dimension at all code levels. This is particularly difficult in that formal grammar rules do not account for actual speaking performance in any but the most formal situations. Grammar rules are a part of the rules of co-occurrence in that they specify one set of those rules for language structure with a formal style (or standard language form). As such, grammar rules tend to be similar to what Thomas Kuhn (1970) calls a theory of normal science in that grammar rules provide a standard body of assumptions for explaining language relations and, in so doing, essentially limit alternative assumptions. Co-occurrence rules provide such an alternative. But any attempt to formalize co-occurrence rules must then start out from nigh onto scratch with the ultimate goal of working itself up to the level of being able to figure out those rules governing the kind of but not overly subtle style shifts in this sentence.

Co-occurrence rules draw both their utility and their confusion from their social dimension. A code switch, which is defined as switching to another set of co-occurrence rules, is only important insofar as the switch is recognizable and socially meaningful in the situation. The borrowed word, "giobba," which created a problem in the interference perspective, is clearly an example of a word tied through co-occurrence rules to a social context in that it developed in response to that context. The example of the reverend "grabbing" a chippendale indicates this further in that men of the cloth and fine furniture can go together in our assumptions about social structure, while grabbing goes more with a stool or an orange crate coupled with a lower status guest. In maintaining these distinctions between speech forms and social structure, we mark the contexts of that structure as significantly "different" or "unique" and concomitantly reconstruct that difference every time we switch styles.

In her discussion of co-occurrence rules, Ervin-Tripp emphasizes the role of social norms in their maintenance. This position is in keeping with the social context basis of those rules in that it makes switches in and out of them meaningful to the participants in those contexts. It nevertheless ignores the fact that co-occurrence rules are nothing more than surface markers of meaningful speech forms and that first there must be some criteria for the application of that meaning to the context. A member of the social context must first know how to assign meaning before he can mark that assignation through 
the shared body of co-occurrence rules. Cicourel (1970) refers to this process of assigning meaning as "interpretive procedures," arguing that the internalization of norms and the knowledge of when and how to apply them to everyday social situations are separate (Cicourel, 1970: 139). The norm that states, "one does not tell a reverend to 'take a load off'," presumes an interpretation of "reverend" and the act of "taking a load off" as both would occur in meaningful social contexts. That interpretation would then search for an overlap (if any) in the possibility of assigning meaning as a mode of determining the suitability of applying the norm. If no overlap can be assigned (as in the case of the doctor's interpretation of the patient's response), some form of a deviant label can (and probably will) be invoked. Just as interesting, however, is the case where considerable overlap is assigned; where the total configuration of co-occurences in speech can be related to the context:
A: What is your name?
A: Pardon me, but I don't believe I've been introduced to you.
B: John.
B: I'm John Smith from the credit division.

B's interpretation that the second example requires more of a display is the logical result of learning language and social structure simultaneously. A dictionary and grammatical breakdown of A's questions would only begin to explain the communication and comprehension of social structure exhibited here. A interprets the context and marks that interpretation through speech. B re-interprets (decodes) the mark, compares it with his own interpretation of the context, and arrives at an assignation of meaning which he then marks in his speech forms. Presumably, the cultural phenomenon known as the "conversation" might then ensue in both cases, but probably very different conversations due to the different definitions of the situations which both speakers have arrived at and marked in one statement each.

The processes through which we understand speech become very complicated very quickly as we try to understand that understanding. As Gumperz and Hymes (1972) remark:

The initial observation is quite like that of grammar: We understand sentences rapidly and unreflectingly in terms of relationships that are not overtly expressed. There must, therefore, be implicit kinds of knowledge in terms of which this ability can be explained. (Gumperz and Hymes, 1972: 325)

That implicit knowledge is a sharing in the ability to interpret social structure. At least in terms of function, then, this ability to interpret is not unlike Chomsky's "deep structure" in that it recognizes a common interpretive competence of members in the social structure. It is, in fact, this interpretive competence that makes them members in the first place; it gives them the potential for taking the surface markers of speech and weaving those markers into a socially meaningful whole.

An additional example might make this point more clear. The following two sentences are from a two-year-and-nine-month-old girl's story:

"The baby cried. The mommy picked it up."

Harvey Sacks (1972) wonders why, for instance, we all hear it to be the mommy of the baby that picks it up since there is no genitive marker (such as "Her mommy ..."). He also wonders why we all hear that sentence two represents an occurrence 
that is explained by a first occurrence represented by sentence one. In short, we all hear a plausible description. Sacks expands upon this:

We hear that it is the mommy of the baby who picks the baby up because she's the one who ought to pick it up, and (you might eventually add) if she's the one who ought to pick it up, and it was picked up by somebody who could be her, then it was her, or was probably her. While it is quite clear that not any two consecutive sentences, not even any consecutive sentences that report occurrences, are heard, and properly heard, as reporting that the occurrences ought to occur in that order, and if there is no information to the contrary (such as a phrase at the beginning of the second, like "before that, however"), then the order of the sentences indicates the order of the occurrences. And these two sentences do present the order of the occurrences they report in the proper order for such occurrences. If the baby cried, it ought to have started crying before the mother picked it up, and not after. Hearing it that way, the second sentence is explained by the first; hearing them as consecutive or with the second preceding the first, some further explanation is needed, and none being present, we may suppose that it is not needed. All of the foregoing can be done by many or perhaps any of us without knowing what baby or what mommy it is that might be being talked of (Sacks, 1972: 330-1).

Besides illustrating how unwieldly such a full description is due to our everyday practice of letting it go unsaid, Sacks is also illustrating what he calls the "fine power of a culture" which does not "merely fill brains in roughly the same way [but] fills them so that they are alike in fine detail" (Sacks, 1972: 332). This well entrenched assumptive world remair:s out of sight most of the time as it is seldom problematic to the members who share it. It is, however, the means through which they interpret the world and, for purposes directly at hand, the means through which they make sensible the world of speech and marked shifts within it.

As we make sense of speech at the level of recognizing descriptions, so also we make sense of subtle style shifts through common interpretations of co-occurrence rules. Consider the following statements from a somewhat eclectic musician:

(1) I was playin' some country tunes last night.

(2) I was playing Mozart last night.

We all accept the fact that in some significant ways, Mozart is considerably different from country music. We treat the two music forms differently through a variety of parallel associations. One of these associations is that country music tends toward the casual while classical music tends toward the formal. Hence, the act of "playing" the two types is, in fact, two different acts and the verb is marked accordingly in each case. It could be further argued that the "playin"" of country music and the "playing" of Mozart are two different words insofar as a word can be defined through the limitation of its applications. Consider a third example:

(3) I was pickin' some country tunes last night.

With this new verb in the context, we would practically never expect to hear it pronounced "picking" as it always co-occurs with only certain "types" of music. It could further be argued that "pickin" " and "playin" " in this context are far closer to being the same verb than "playin" "and "playing" across contexts. This need not be a major issue, however, for as it has been 
defined, a style shift carries a new meaning in itself, and it would seem hardly likely that any lexical items retained would not be colored in the shift. The shift here occurs with a shift of context and, appropriately, of meaning. Hence, that which we all know about the difference between classical music and country music is recognized and maintained through style shifts.

Placing our musician back into the classical music context, it is possible to imagine a musically critical neighbor (who had heard Mozart treated far better) asking the musician just what he thought he was doing. The musician then has at least two choices for styles. He can choose to be humble through reducing his status through style shift:

(4) Well, I was only tryin' to play Mozart.

The shift is marked by altering the pronunciation of "trying" to "tryin" " (which, among other things, is a status marker), and through a slight emphasis on "try" and "Mo" which marks a culturally defined "whine." Or should the musician be a little more annoyed and assertive to the point that he might wish to challenge the remark of his neighbor, he could respond with:

(5) Well, I was TRYing to play MOzart.

This shift places far more emphasis on "try" and "Mo" (the emphasis will usually co-occur in such a parallel fashion), and this emphasis co-occurs with a return to the more formal pronunciation of "trying." This overall co-occurrence also co-occurs with the social dimensions of status and, significantly, the classical music in which our musician is now claiming an ability through his style shift.

If it is possible to talk about shared perceptions of co-occurrence rules as markers of style shifting, it should also be possible to talk about some degree of permanence of codes within a population. Labov (1970) states that it is only possible to speak of language varieties and alternation when some set of co-occurrence rules has been established. In this sense, co-occurrence rules become used as a yardstick for measurement, and any reliable measurement tool should not be fluctuating across time and situations. Having set such a yardstick himself with regard to Black English and Standard English, Labov then points to a very short explanation provided by a twelve-year-old Negro boy who switched between the two codes (as measured by co-occurrence rules) eighteen times (Labov, 1970: 35). Labov asks, "Where and how do stylistic meanings enter into this process?"

The social dimensions of co-occurrence rules have already been outlined, and if this dimension in fact exists, it should be possible to isolate relations between the social context of speaking and the style of the speech. In his sample of eighteen code shifts, Labov feels at a loss to find this relation. This brings up the question of language variants as examples either of code-switching or of free variation. Can it be said on the one hand that a variant represents a significant shift of code, and, on the other, that the variant is random and has no significance at all? While it would seem that all elements of social context remained constant during Labov's eighteen shift sample, it is nevertheless possible to imagine a social context which is essentially incongruous for the speaker (such as one containing an interviewer with a tape recorder) and to which standard assumptive rules do not apply. A mixed style might well be the result of a mixed speech situation in which immediate responses to speech forms (as pointed out by Sacks) were in some way interfered with, thus not permitting the flow of 
implicit knowledge upon which speech styles depend. Having mentioned this dependence numerous times, a discussion focusing on the nature of speech situations is long overdue. This discussion of the second major theoretical obstacle mentioned earlier will be guided by the concept "domain" (Fishman, 1967, 1971; Fishman, Cooper and Ma, 1971; Cooper, 1969).

\section{Language Domain}

'Proper' usage dictates that only one of the theoretically co-available languages or varieties will be chosen by paricular classes of interlocutors on particular kinds of occasions to discuss particular kinds of topics (Fishman, Cooper and $\mathrm{Ma}, 1971:$ 583)

In short, there must be a study of speaking that seeks to determine the native system and theory of speaking; whose aim is to describe the communicative competence that enables a member of the community to know when to speak and when to remain silent, which code to use, when, where and to whom, etc. (Hymes, 1967: 13).

A theory of speaking (or proper usage) posits a dual knowledge on the part of every speaker-a knowledge of language and a knowledge of when, where, how, and to whom to use it (Fishman, 1965). It is also assumed that these two forms of knowledge are in part defined in terms of each other. A knowledge of language would be meaningless unless grounded in some socially meaningful communication. Similarly, it is difficult to make that communication meaningful without a knowledge of language. Learning of society and of language occur con-currently, and necessarily so. Hence, it is impossible to talk about speaking without knowing something about the way in which the situations of speaking have been defined as situations by the speakers as they learn and use language within them (Gumperz, 1968: 381).

It is in response to this issue that the notions of speech community and speech situation came about. A speech community refers to those communities which share "both rules for the conduct and interpretation of acts of speech, and rules for the interpretation of at least one common linguistic code" (Hymes, 1967: 18). One could add, in line with Labov's (1972) comment on single style speakers, that there will always potentially be more than one common linguistic code in anything that could be called a speech community. Nevertheless, the definition of speech community does not necessarily specify a dividing line for determining when one has stepped out of the community. Rules concerning "conduct and interpretation of acts of speech" are not set in time nor across members of what might seem to clearly be one community. Similarly, there will likely be at least partial adherence to those rules among those who seem clearly outside the community and a partial adherence to rules outside the immediate community by those inside. The provision that any group which exhibits "linguistic peculiarities" may be treated as a speech community (Gumperz, 1968: 381) is only a good working definition insofar as the members of such a community are seen as having the potential of holding simultaneous membership in other communities. An occupational association, for instance, might likely exhibit linguistic peculiarities, but in terms of the social dimensions of that association, other speech styles that the members use in other situations (and their perceptions of those styles and situations) would certainly be an important factor. 
The notion of situation (which can cut across certain speech communities) refers to "any constellation of statuses and setting which constrains the interaction that should or may occur. . A situation, like a status, is a cultural unit, so that ethnological study is necessary to determine classes of situations" (Ervin-Tripp, 1971: 50). Hence, a situation, as a cultural unit, is constructed by the participants through cultural tools. Not surprisingly, therefore, situations are marked by language forms. As Blom and Gumperz (1972) noted in their study of code-switching in Norway, the language forms that mark situations are also means for re-marking them, resulting in a new situation. A code-switch, in itself, is enough to instantly communicate one member's redefinition of the situation, forcing the listeners to discard the previous congruence between language and social context in their search for a new congruence (if any can be found) with the new language form.

An additional cultural unit is the topic of communication and, like speech community and situation, it is part of the whole speech context and must be understood in terms of it. Topics are only important in the study of language insofar as the members perceive themselves to be changing them from time to time. We conceive of ourselves as talking about things when we talk, and those "things" are often labeled as such. More importantly, we label topics in terms of situations and in terms of groups of interlocutors. We also distinguish topics through language forms in that we learn about the possibility of constructing a topic terms of groups of interlocutors. We also distinguish topics through language forms in that we learn about the possibility of constructing a topic through those language forms in context. The more obvious cases of this include individuals who receive specialized training in a "topic" and are unable to communicate about it in any but the language forms of that training. We also learn to socially construct our most intimate feelings (a topic) into communications through language forms, and alternative language forms may well seem wholly inappropriate (Fishman, Cooper and Ma, 1971: 585). It can be said, therefore, that a topic is, in part, both defined and understood in terms of the language form through which it is communicated. Hence, a discussion of woman's rule in the English language presupposes a social context within which both the role and the language operate, and the "topic" has no direct translation outside of that configuration. In this sense, then, topic is not so much an explanation of differential use of language forms but rather part of the overall set of co-occurrence rules among those forms. It is the outcome of assigning meaning to those rules.

The combination of speech community, situation and topic into an overall perspective results in the concept of "domain." Domain has been defined as "a socio-cultural construct abstracted from topics of communication, relationships between communicators, and locales of communication, in accord with the institutions of a society and the spheres of activity of a speech community" (Fishman, Cooper and Ma, 1971: 587). Domains are simultaneously defined in terms of societal institutions and the individual process of constructing those institutions in everyday life. Put more traditionally, it is a concept both of sociology and of social-psychology. As a concept, it recognizes the complexity of the speech act in a social context through pointing out the internal coherence of all the elements involved in communication. Like all concepts with noble aspirations, however, it is somewhat difficult to get a handle on, and much of this difficulty stems from the attempts of the concept to account for the place of role relationships in speech.

Fishman, Cooper and $\mathrm{Ma}$ (1971:570) have tried to describe these role relationships in terms of open and closed networks. A closed network refers to those role relationships "governed by a single, overriding, fully formed set of specifications" (Fishman, Coopera and Ma, 1971: 569). An open network is the corresponding ideal type at the other end of 
the continuum in which these specifications are open and fluid. The assumption for language concerns is that the more a role relationship is specified, the more the language forms utilized will be specified. Since role relations are fundamental to the concept of domain, the degree to which those relations vary will in large part differentiate domains.

Networks describe or characterize kinds of role-relationships along the dimension of permissible role fluidity. Individuals who have experienced great danger together, or great intimacy, may permit no other considerations to govern their future relationships. Individuals who stand poles apart in status, rights, and obligations may be similarly fixed and unalterable in their relationships. However those who neither partake in a common, intensive past, nor in a current overriding difference may range over a number of similarities and differences in the course of a particular encounter without definitely leaving the middle range of role-relationships. (Fishman, Cooper and $\mathrm{Ma}, 1971: 571$ )

As is clear from the above, however, attempts to differentiate domains in terms of role relationships inevitably encounter the additional distinction of status.

John Gumperz (1964) has suggested that role relationships be further distinguished in terms of personal and transactional interactions, referring respectively to the absence or presence of status distinctions. Combining this dimension with that of network, it then becomes apparent that the two types of closed network mentioned in the above quotation are respectively personal and transactional interactions within one network and within which there should rarely be much shifting between interaction types. Conversely, an open network (defined as having no overriding specifications) would be much more likely to vacillate between interaction types (or along the continuum) as definitions of the situation would be open for immediate construction. It is in these situations that one would expect to find more rapid code-switching and this would account, in part, for Labov's dilemma concerning the rapid Black English-Standard English code-switching of the twelve-year-old boy. The import here is that clear-cut maintenance of language forms depends upon the degree to which their domains contain closed networks of role relationships. Linguistic repertoires are direct reflections of role repertoires, and distinct language varieties are dependent upon the degree of role compartmentalization (Fishman, 1967: 32). As that compartmentalization changes into a more open network, the "situation switching" function of a change in language form becomes more of a "metaphorical switch" through which the definition of the situation is not assaulted but rather placed in a temporarily different light for the participants (Blom and Gumperz, 1972: 424-425). If a discrepancy exists as to the openness of the network among the participants, there will be a corresponding discrepancy concerning the importance of shift in language form. Such a situation would occur when particular role relationships were in the process of changing lor fluctuating) between open and closed networks. The importance of language forms as markers is dependent upon the construction of situations by the participants; hence, an alteration of those constructions also, by definition, alters that which is marked by language forms and therefore alters the meaning of a shift in those forms.

In order to account for the differentiation of the roles of language forms within a society, Charles Ferguson (1959) introduced the term "diglossia." Diglossia refers to those instances of bilingualism where two language forms exist side by side in society, each having distinctly different relations to the social structure. He referred to them as the $H$ ("high") variety and the $L($ "low") variety, the former being the official, formal, learned from having a basis in the society's major 
institutions, and the latter being the informal, intimate form (often labeled dialect) having a basis in the home. Those members of the society who interact in both spheres (such as the earlier case of the Belgian businessman) would then have a facility in both language forms, but only insofar as that form related to its domain of use. It is in this sense that language forms are said to be functionally related to social structure (Hymes, 1967: 43).

The concept of diglossia assumes a stratified society. While the specification of $H$ as "high" and $L$ as "low" was later dropped (see Fishman, Cooper and Ma, 1971: 566), the implication is clear-that language forms (whether recognized languages or not)discriminate status distinctions. A "monolingual" stratified society accomplishes the same end through varieties of its one language. To extend Ferguson's definition, any society that is stratified will be diglossic in the sense of having differential access to codes and subsequent differential use of them across people and across situations. Returning now to the personal-transactional distinction made by Gumperz, a personal transaction would take place in some $L$ variety, and a switch to a transactional interaction would be marked by the use of some $H$ variety. The former carries connotations of intimacy and the latter carries connotations of status. Adding Fishman's notion of closed vs. open networks of role relationships, the earlier assertion that closed networks maintain language forms gains support. The more that the domains of the $H$ and the $L$ variety are separate (through role specification of a closed network), the more each variety becomes associated with all other characteristics of those domains, and the more intensely each variety strongly carries connotations of status and intimacy respectively. Each language form then represents an extremely large body of social information, and the forms will be maintained as long as it is necessary to convey that information. As the domains alter (or overlap), the information conveyed by the language forms will also change.

Access to the $\mathrm{H}$ variety becomes an important variable in describing those groups with little access to the domains of that variety. In a situation of diglossia, access to a language form which marks status will be restricted in much the same way as access to the power and resources which accompany that status. An individual with no ability in speaking the $H$ variety will likely remain at the bottom of the status hierarchy and, conversely, most individuals at that bottom will reflect that inability. This assertion is derived from the assumption that language forms co-occur with domains, and that an inability to actively participate in a domain will result in a lack of knowledge in those language forms. Hence, confrontations with the $\mathrm{H}$ variety will occur with transactional interactions in closed networks where the low status individual is given orders in a language form in which he cannot fight back. Such is the experience of whole groups of individuals who, as groups, are maintained in this relation to the social structure. The lower in status the group, the more closed its networks, and the more significant the distinctions in language forms in differentiating domains of intimacy and status.

This point is punctuated by an example drawn from the bilingualism of Chicanos living in the United States. One of the standard style shifts occurs in Standard Spanish with the disciplining of children. Spanish contains a choice of intimate/polite in the second person singular (tu/usted) and this choice is reflected in the corresponding conjugation of any verb. A parent almost always uses the intimate form with his child as, for instance, ven aqui (come here). If, however, the child does not obey, a style shift may ensue as with an English speaking parent, but whereas English must resort to other means ("Come here this minute, Thomas Edward Smith III"), Spanish can simply switch all forms to the polite conjugation (venga aqui) and convey the same message. With Chicanos living in the United States, however, Gumperz (1972) notes that ven aqui becomes, 
"come here, you," in place of venga aqui, suggesting that in this country, the imperative in English far surpasses any other possibility in Spanish for getting immediate action. In light of the status positions of English language domains from the perspective of many Chicanos, it is not surprising that English is the language for commands. Furthermore, considering that the major English language domain for most Chicano children in the classroom, it is also not surprising that such a style shift by their parents brings results.

A further extension of the domain perspective sheds some light on problems of bicultural/bilingual education. The "stilted" and meaningless English language exercises noted by Wax, Wax and Dumont (1964) in the Indian classroom and by Gumperz (1972) in the Chicano classroom understandably do not work in that they have no relation to within-domain communication in either culture, not to mention their lack of relation to the student's domains. The result of this activity is to create a foreign domain to both cultures and to generate sets of meanings and language forms with relevance only to it. Hence, the old argument that going to school teaches one only how to go to school becomes an even more relevant statement with regard to the lower status (" $L$ " speaking) child in what may well be his first encounter with the higher status ( $H$ ) language form. As Gumperz (1972) also noted, the same teacher confronted by children who are good speakers of Standard English immediately relaxes and approaches a normal speaking routine. It is through this "normal" speaking routine that social structure and language become sensible to the participants. As Susan Philips (1972) concluded from her attempts to understand the failings of Indian education, the learning of a language does not necessarily include learning the cultural rules for its use. Borrowing Gumperz's notion of status situated language forms, it could be appended here that those cultural rules will not be learned unless the learning individual participates in the relevant domains to their use. An individual systematically denied access to the domains of formal Standard English is, by definition, systematically denied access to those language forms, and it is doubtful he could acquire them in a simulated domain playpen.

The perspective of a closed network as maintaining these boundaries for status marked language forms has, as mentioned above, the opposite effect of also maintaining boundary markers for within-group intimacy. The specification of language forms by status results in alternative bodies of co-occurrence rules which convey information of status to both outsiders and to insiders (Williams, 1970: 383; Ervin-Tripp, 1971: 39; Blom and Gumperz, 1972: 418-19). Borrowing an idea from labeling theory, just as language forms (and other markers) allow the "respectable" world to recognize "deviants" in their midst, so also do those same forms allow those "deviants" to recognize each other. Such recognition permits the immediate marking of outsiders who are almost, by definition, higher in status and a potential threat. In opposition to some of the linguistically based cultural deprivation theories (see Bernstein, 1966, 1968; Olim, 1970), some of the greatest emphasis on skill in the verbal arts has been encountered among poor, inner-city speakers of Black English (Gumperz, 1972: 186). It would seem a sensible assumption that the ability to recognize style variation in language would have some relation to the social need for possessing that ability.

Should the above status distinctions begin to break down, whether for an individual or for a group, there would be a significant language shift (Fishman, Cooper and Ma, 1971: 569). As explained earlier, such an alteration in status distinctions is a shift from a closed network to an open network. In terms of domains, this would force the previous distinctions among language forms into a domain overlap. At the recognized language level, Fishman (1971) has traced the acculturation of 
immigrant groups in terms of this overlap. As members of these groups enter into the new dominant culture, the language of that culture begins to invade the domains of the immigrant language. By the time this dominant language invades the domains of intimacy (which is usually several generations), the immigrant language has lost its last major domain stronghold and is likely to disappear from all but the most ritualized activities (Fishman, 1971: 306). This perspective also makes the co-ordinate-compound bilingual distinction clear in pointing to the con-current shift from the former to the latter as domain overlap occurs. Co-ordinate bilingualism is maintained through domain segregation, this segregation providing the parallel meaning systems which characterize it. As the segregation breaks down, the bilingual becomes either a compound bilingual or (as is more common in the United States), a monolingual. Those languages which have survived in the United States are either those locked into closed networks of role relationships, such as Spanish or the Indian languages, or those that have consciously maintained language domains, such as Yiddish (see Fishman, 1966).

Coming full circle, it should be clear at this point just why the Italian-American loan-word giobba does not apply to employment alternatives in Italy. It should also be clear why several generations of filling "giobbas" in the United States renders Italian irrelevant for making the immediate environment sensible. The initial interest of linguists in language interference has placed language concerns firmly in a social context and has brought into question the basic assumptions which generated the interference perspectives in the first place. While it has not been the sole purpose of this discussion to illustrate a theoretical patricide, it is nevertheless useful to trace the relationship between the types of questions being asked about phenomena and the types of assumptions that underlie them. With regard to language concerns, the fields of linguistics and sociology are beginning to experience a domain overlap, and they may both encounter difficulty in maintaining the colorful artifacts which have marked their respective cultures.

\section{Appendix}

Notes on Methodological Procedures Not Discussed

in the Body of the Paper

\section{Linguistic Focus}

The possession of a magnetic tape recorder and a willingness to press its "on" button in the most unlikely natural settings do not insure that the researcher will procure the kind of language data he is looking for. An emphasis on the study of speech rather than on language (or of performance rather than competence) requires natural speech data, but the acquisition of this data requires some knowledge of speech at the outset. The question, "Why does anyone say anything?", must already be in the form of a working hypothesis.

Perhaps the most immediate problem is what Labov calls the "observer's paradox" - "to obtain the data most important for linguistic theory, we have to observe how people speak when they are not being observed" (Labov, 1972: 113). As with all forms of field research, so also must the linguist find some way of approaching his subject without overly altering his behavior. In the case of the one-to-one interview, Labov suggests the use of topic change, thus making use at the outset of 
a dynamic inherent in what he studies. Labov has found that topics relating to (1) death and violence, (2) sex, and the rituals thereof, and (3) moral indignation, all provide excellent opportunities for the speaker to become involved in his speech to the point of forgetting (somewhat) the interviewer's presence (Labov, 1972: 114). Another possibility along this line includes directing the subject to a discussion of language itself, which on the one hand provides justification for using a tape recorder (particularly if the researcher is "learning" a second language), but on the other hand focuses the subject's attention on the subject of study itself and will certainly result in a more careful speaking style.

In the interview form, perhaps the most difficult subject is the child. Particularly with a fairly young child, the only practical use of the recorded interview would be with a focus on some form of play activity. In this way, the relationship between the interviewer and the child would approach some semblance of normality from the child's perspective and might produce the more natural elicitations so often absent from more formal child-adult interactions. It is interesting to note that the provision of a reasonable setting for verbal interaction (the assumption of Labov's topic change as well) assumes that which is the object of a sociological study of language use. Using several types of methodology con-currently is therefore not only possible, it is also quite preferable.

The use of tasks in the linguistic interview provides a number of speaking styles for interpretation. The above mentioned "naturalistic" interview is designed to provide samples of casual speech. There are times, however, when the interviewer may well want samples of a more "careful" speech (whatever that entails in a particular speech community) and will want to know how to make the subject more aware of his speech. This is conceivably not a great problem. Additional care in speech style (primarily used for phonological study) can be initiated through asking the subject to read some sample of discourse and, beyond that, lists of words. In The Social Stratification of English in New York City, Labov utilized these techniques to isolate particular phonemes which varied regularly across class levels and across speaking styles within class levels (Labov, 1966). Variants of this methodology can, of course, also be used for linguistic structural analysis.

An alternative route to naturalistic speech (particularly useful with children) consists of recording groups of subjects at the same time. It is hoped here that the presence of a number of people will introduce at least some elements of normal speech domain as they respond to each other instead of to the interviewer (see Blom and Gumperz, 1972). While it introduces the possibility of a "group performance" for the benefit of a probably out-group researcher, it nevertheless offers the potential of individual involvement in communication. This method is, of course, of less utility for phonological analysis due to technical problems of obtaining audible recordings from all members coupled with the inevitable background noise.

The group membership of the interviewer is, as mentioned above, an important variable. If he is an outsider to the speech community, his speech will no doubt reflect it. Even an artful interviewer capable of covering this might still be classed an outsider because of social giveaways through other than language behavior. On the other hand, using a member of the speech community as an interviewer opens an alternative can of worms through introducing his previous relationship and subject's perceptions of future relationships with him into the picture. In short, there is a clear advantage to having both insiders and outsiders in the task (Labov, 1972: 115). 


\section{Sociological Focus}

The determination of language domains and their elements is in large part a task of participant observation (Cooper, 1969: 196; Fishman, Cooper and Ma, 1971: 589). Any given speech community will have different physical settings, types of activities, and perceptions regarding the whole configuration of language related behavior (see earlier discussion). The observation of this structure as a participant can provide numerous insights as well as avoiding obvious errors in projecting domain upon a community. In particular, the most significant domains in a community are often conscious topics of conversation. Informants' statements regarding the people they encounter, where they encounter them, and the nature of their relationships with them can focus the researcher's attention not only upon well-known community domains and differential participation in them, but also upon those domains (including the researcher's projections) which could exist but do not. This latter case might include various forms of voluntary associations which don't exist at all in the community (such as a nonexistent teacher-parent association) or domains of another speech community "potentially" open to the community under study (such as non-used welfare office). Particularly in the case of a bilingual/bicultural communtiy, the question of which domains overlap across cultures and languages and which do not is significant at the outset. The degree of this overlap and the differences between overlapping and non-overlapping domains for each group are likely to not be readily apparent and must be determined empirically before they can enter into a more general sociological interpretation as variables.

A method for breaking down the elements of domains consists of analyzing "folk terminology" regarding speech situations and topics (Ervin-Tripp, 1971: 51-4; Fishman, 1971: 255). As suggested above, domains are conscious in group interaction. One domain element such as a speech situation may well be waiting pre-packaged for the researcher in the local lexicon. Examples such as "church service," "hamburger stand," "school yard," and "pool hall" may seem obvious places to look, but it may turn out there is a significant difference between the "front room of the pool hall" and "the back room of the pool hall," and these distinctions may be proffered to the researcher through conversation. A similar phenomenon occurs with topics, such as "we were just telling jokes" as an explanation by the participants of what they were doing. The perception of topic in conversation is an abstraction from interaction, and a double-checking of these abstractions through common labels prevents jumping to conclusions in the over-anxious formation of a variable. Once again, however, with regard to both situations and topics, an important factor will be those that do not exist. To borrow the above example for a slightly different purpose, the absence of the topic, "the discussion of welfare benefits," among those eligible to receive benefits is worth noting.

Domains are subject to being validated (and ultimately compared) through other forms of methodology. Fishman, Cooper and $\mathrm{Ma}$ (1971) organized data on language use collected across what they hypothesized to be several language domains. This included information on which languages were used where, coupled with various measures of language ability. Many of these measures were not too dissimilar from Macnamara's (1967) Flexibility Tests which are sensitive to domain differentiation. A subsequent factor analysis of this data resulted in factors which matched the breakdown of domains obtained earlier by participant observation. 
Other language tests sensitive to sociological variables:

Word Frequency Estimation List: (Fishman, Cooper and $\mathrm{Ma}, 1971$ ) This test includes lists of words drawn in equal numbers from various situations which exist in the community. The subject is asked to rate the frequency with which he hears them (more than once a day; once a month, etc.). The "same" words occur in each list but in different languages. [A version of this test could be used in a monolingual setting or as an addition to a bilingual setting through breaking down styles within languages and providing a rough set of synonyms across styles.]

Word Naming: (Fishman, Cooper and Ma, 1971) Related to the above, speech situations are presented with the request that the subject produce as many words in each language as possible with applications in that situation (kitchen, etc.). As above this is bilingual based. Its possible use within one language might consist of differentiating styles from which lexical items are drawn as a measure of style shifting's relation to situation. Also, as above, this style differentiation could be compared across two or more languages.

Domain Completion Tasks: (Fishman, Cooper and Ma, 1971) The subject is presented with a few elements of a domain and is asked to complete it. For example, "You are talking with a school friend about homework. Where are you talking?" and varieties of this sort ask the subject to make the situation sensible. As a test of domain, two presented elements can be incongruous ("You are talking with your Priest at the racetrack") in an attempt to find out how the subject would make the situation manageable. Fishman, Cooper and $\mathrm{Ma}$ (1971) found that incongruous situations were usually filled out with a domain element from either of the two presented-seldom from a third.

Word Association Tests: (Entwisle, 1968, 1970) Generally designed for children, this test consists of a series of one word stimuli accompanied with the request that the subject say the first word that comes into his mind. This test is a measure of language development (as children learn the word classes) and is also simultaneously a measure of the semantic reality of the child. An example of this latter case might be the stimulus word "sour" for which Entwisle (1970) received "bad" and "rotten" in the suburbs and "okay" or "still good" in the inner city.

Semantic Differential: (Osgood, Suci and Tannenbaum, 1957; Hymes and Bittle, 1967; Luhman, 1973) The semantic differential can be used as a measure of concepts' structures as well as empirical validation of basic word classes for a particular community (see Hymes and Bittle, 1967; and Cole, Gay, Glick and Sharp, 1971).

Homonym Differentiation: (Luhman) Based on the homonyms present in English, most pairs vary in frequency of occurrence (such as "bear" and "bare"). They also vary as parts of speech. The above pair is a noun/adjective combination while "hare" and "hair" are both nouns. A story for children composed of such homonyms would act as a measure of sentence comprehension in that the use of the less common (for children) "bare" would clearly not be the animal to any 
child who could recognize adjective placement as a marker of meaning. [This is of particular interest with regard to Spanish and English where adjectives are placed after the noun and before the noun respectively.]

In addition, personal information that may have bearing upon language use ('Where were you born? What newspapers do you read? In what languages?" etc.) can be gathered through standard questionnaire techniques, either in the form of an interview or in a written form (see Fishman, Cooper and Ma [1971] for extensive use of both). In the case of an unknown bilingual community with unknown rates of illiteracy, the former approach is mandatory. Otherwise, there is the standard consideration of flexibility vs. quantity respectively.

\section{References}

Barker, George C.

1947 "Social functions of language in a Mexican-American community." Acta Americana 5, No. 3: $185-202$.

1950 "Pachuco: an American Spanish argot and its social functions in Tucson, Arizona." University of Arizona Social Science Bulletin, No. 18. Tucson: University of Arizona Press.

Bernstein, Basil

1966 "Elaborated and restricted codes: an outline." Sociological Inquiry 36:254-6.

1968 "Some sociological determinants of perception-an inquiry into sub-cultural differences." In J. Fishman (ed.), Readings in the Sociology of Language. New York: Humanities Press.

Blom, Jan-Petter and John Gumperz

1972 "Social meaning in linguistic structures: code-switching in Norway." In Gumperz and Hymes (eds.), Directions in Sociolinguistics. New York: Holt, Rinehart and Winston.

\section{Carrow, Sister Mary Arthur}

1957 "Linguistic functioning of bilingual and monolingual children." Journal of Speech and Hearing Disorders $22: 371-380$.

\section{Chomsky, Noam}

1965 Aspects of the Theory of Syntax. Cambridge: M.I.T. Press.

Christian, Jane Macnab and Chester C., Jr.

1966 "Spanish language and culture in the Southwest." In J. Fishman (ed.), Language Loyalty in the United States. The Hague: Mouton.

\section{Cicourel, Aaron V.}

1970 "The acquisition of social structure: towards a developmental sociology of language and meaning." In Jack Douglas (ed.), Understanding Everyday Life. Chicago: Aldine. 
Cole, Michael, John Gay, Joseph A. Glick and Donald W. Sharp

1971 The Cultural Context of Learning and Thinking. New York: Basic Books.

Cooper, Robert

1969 "How can we measure the roles which a bilingual's languages play in his everyday life?" In L. G. Kelly (ed.), Description and Measurement of Bilingualism. Toronto: University of Toronto Press.

Entwisle, Doris R.

1968 "Subcultural differences in children's language development." International Journal of Psychology 3:13-22.

1970 "Semantic systems of children: some assessments of social class and ethnic differences." In F. Williams (ed.), Language and Poverty. Chicago: Markham.

\section{Ervin-Tripp, Susan}

1971 "Sociolinguistics." In J. Fishman (ed.), Advances in the Sociology of Language. The Hague: Mouton.

Ervin, Susan and C. E. Osgood

1954 "Second language learning and bilingualism." Journal of Abnormal and Social Psychology 49(supplement):139-146.

Ferguson, Charles $\mathrm{A}$.

1959 "Diglossia." Word 15, No. 2:325-340.

Fishman, Joshua A.

1965 "Who speaks what language to whom and when?" La Linguistique 2:67-88.

1966 Language Loyalty in the United States. The Hague: Mouton.

1967 "Bilingualism with and without diglossia; diglossia with and without bilingualism." Journal of Social Issues 23, No. 2:29-38.

1971 Advances in the Sociology of Language. 2 vols. The Hague: Mouton.

Fishman, Joshua A., Robert L. Cooper and Roxana Ma

1971 Bilingualism in the Barrio. Bloomington, Indiana: Indiana University Press.

Gumperz, John J.

1964 "Linguistic and social interaction in two communities." In Gumperz and Hymes (eds.), The Ethnography of Communication-American Anthropologist 66, No. 6, part 2.

1967 "On the linguistic markers of bilingual communication." Journal of Social Issues 23:48-57.

1968 "Speech community." In David Sills (ed.), International Encyclopedia of the Social Sciences. Vol. 9. New York: Macmillan and Free Press.

1969 "How can we describe and measure the behavior of bilingual groups?" In Kelly (ed.), Description and Measurement of Bilingualism. Toronto: Univeristy of Toronto Press. 
1972 "The communicative competence of bilinguals: some hypotheses and suggestions for research." Language in Society $1: 143-154$.

Gumperz, John J. and Dell Hymes

1972 Directions in Sociolinguistics. New York: Holt, Rinehart and Winston.

Hasselmo, Nils

1969 "How can we measure the effects which one language may have on the other in the speech of bilinguals?" In L.

G. Kelly (ed.), Description and Measurement of Bilingualism. Toronto: University of Toronto Press.

Haugen, Einar

1956 "Bilingualism in the Americas: a bibliographic and research guide." American Dialect Society 26.

1958 "Language contact." Proceedings of the Eighth International Congress of Linguists. Oslo: Oslo University Press, 771-785.

1965 "Bilingualism as a goal of foreign language teaching." On Teaching English to Speakers of Other Languages, Series I. Champaign, Illinois: National Council of Teachers of English.

Hymes, Dell

1967 "Models of the interaction of language and social setting." Journal of Social Issues 33, No. 2:8-28.

1967 "Why linguistics needs the sociologist." Social Research 34, No. 7:632-647.

Hymes, Dell and William Bittle

1967 Studies in Southwestern Ethnolinguistics. The Hague: Mouton.

Kuhn, Thomas

1970 The Structure of Scientific Revolutions. Chicago: University of Chicago Press.

Labov, William

1966 The Social Stratification of English in New York City. Washington D.C.: Center for Applied Linguistics.

1969 "How can we describe and measure the behavior of bilingual groups? -Commentaries" In Kelly (ed.), Description and Measurement of Bilingualism. Toronto: University of Toronto Press.

1970 "The study of language in its social context." Studium Generale 23:30-87.

1972 "Some principles of linguistic methodology." Language in Society 1:97-120.

Laffal, J.

1965 Pathological and Normal Language. New York: Atherton Press.

LePage, R. B.

1969 "Commentaries-How can we measure the effects which one language may have on the other in the speech of bilinguals?" In L. G. Kelly (ed.), Description and Measurement of Bilingualism. Toronto: University of Toronto Press. 
Liles, Bruce L.

1972 Linguistics and the English Language. Pacific Palisades, California: Goodyear.

Luhman, Reid

1973 "Type construction and application in social experience." Lawrence: University of Kansas, unpublished Master's thesis.

Mackey, W. F.

1965 "Bilingual interference: its analysis and measurement." The Journal of Communication 15: 239-249.

Macnamara, John

1966 Bilingualism and Primary Education. Edinburgh: Edinburgh University.

1967 "The bilingual's linguistic performance." Journal of Social Issues 33, No. 2.

1969 "How can one measure the extent of a person's bilingual proficiency?" In L. G. Kelly (ed.), Description and Measurement of Bilingualism. Toronto: University of Toronto Press.

Olim, Ellis G.

1970 "Maternal language styles and cognitive development of children." In F. Williams (ed.), Language and Poverty. Chicago: Markham.

Osgood, Charles, George Suci and Percy Tannenbaum

1957 The Measurement of Meaning. Urbana, Illinois: University of Illinois Press.

Philips, Susan

1972 "Acquisition of roles for appropriate speech usage." In Abrahams and Troike (eds.), Language and Cultural Diversity in American Education. Englewood Cliffs, New Jersey: Prentice Hall.

Rayfield, J.

1970 The Languages of a Bilingual Community. The Hague: Mouton.

Sacks, Harvey

1972 "On the analyzability of stories by children." In Hymes and Gumperz (eds.), Directions in Sociolinguistics. New York: Holt, Rinehart and Winston.

Sawyer, Janet B.

1971 "Social aspects of bilingualism in San Antonio, Texas." In Harold B. Allen and Gary Underwood (eds.), Readings in American Dialectology. New York: Appleton-Century-Crofts.

Wax, Murray, Rosalie Wax and Robert Dumont, Jr.

1964 "Formal education in an American Indian community." Supplement to Social Problems 2, No. 4(Spring).

Weinreich, Uriel

1953 Languages in Contact. The Hague: Mouton.

Williams, Frederick

1970 "Language, attitude, and social change." In F. Williams (ed.), Language and Poverty. Chicago: Markham. 